TRANSACTIONS OF THE

AMERICAN MATHEMATICAL SOCIETY

Volume 224, Number 1, 1976

\title{
NONREGULAR ULTRAFILTERS AND LARGE CARDINALS
}

BY

\author{
JUSSI KETONEN
}

ABSTRACT. The relationship between the existence of nonregular ultrafilters and large cardinals in the constructible universe is studied.

1. Introduction. Our notation and terminology follows that of the most recent set-theoretic literature: for example $|x|$ denotes the cardinality of the set $x$, small Greek letters $\alpha, \beta, \gamma, \ldots$ denote ordinals, cardinals are initial ordinals, the Greek letters $\kappa, \lambda, \ldots$ are reserved for denoting cardinals, and so on. Needless to say, the word 'ultrafilter', the central object of our study, refers to a maximal filter. In particular, to simplify terminology, the phrase ' $U$ is an ultrafilter over $\boldsymbol{k}$ ' will always refer to a uniform ultrafilter over $\kappa$, that is, for any set $x$ we require $x \in U \rightarrow|x|=\kappa$.

A central notion in the study of structural theory of countably incomplete ultrafilters over uncountable cardinals is the degree of regularity of the ultrafilter.

1.1. Definition. An ultrafilter $D$ is $(\kappa, \lambda)$-regular if there is a set $S \subseteq D$ of cardinality $\lambda$ such that

$$
T \subseteq S \wedge|T|=\kappa \rightarrow \bigcap T=0 .
$$

An ultrafilter is $\lambda$-regular if it is $(\omega, \lambda)$-regular.

This notion is due to Keisler. He showed that ultrapowers taken using fairly regular ultrafilters have a great model-theoretic significance. Keisler also showed that for every cardinal $\lambda$ there is a $\lambda$-regular ultrafilter over $\lambda$. (For details, see for example Chang and Keisler [2] .) The reverse direction here, namely the existence of suitably nonregular ultrafilters was left completely open. It is obvious that every ultrafilter over a cardinal $\lambda$ is $(\lambda, \lambda)$-regular. Beyond this, our usual set-theoretic axioms do not seem to tell too much. On the other hand, in the constructible universe, K. Prikry [13] has shown that every ultrafilter over a successor cardinal $\kappa^{+}$is $\left(\kappa, \kappa^{+}\right)$-regular. R. Jensen [3] extending the work of Prikry showed that every ultrafilter over cardinals of type $\omega_{n}(n<\omega)$ is regular.

The introduction of large cardinal axioms seems to be the most natural approach to the problem of existence of nonregular ultrafilters.

Received by the editors September 16, 1974.

AMS (MOS) subject classifications (1970). Primary $02 \mathrm{~K} 35$.

Key words and phrases. Ultrafilter, nonregular, weak compactness, weak normality. 
1.2. Definition. A cardinal $\lambda$ is measurable if there is a $\lambda$-complete ultrafilter $D$ over $\lambda$; i.e.

$$
X \subseteq D \wedge|X|<\lambda \rightarrow \bigcap X \in D .
$$

It is then easy to see that every such $D$ is not $(\rho, \lambda)$-regular for any $\rho<\lambda$. The cardinal $\lambda$ here is huge indeed; using ordinary forcing techniques we can produce a weakly inaccessible cardinal $\lambda^{\prime}$ below the continuum carrying an ultrafilter which is not $\left(\rho, \lambda^{\prime}\right)$-regular for any $\rho<\lambda^{\prime}$ (for details, see Prikry [12]). However, the question of Keisler and Gillman, namely the existence of a nonregular ultrafilter over $\omega_{1}$, is completely open. As was mentioned before, Prikry [13] showed that in the constructible universe, every uniform ultrafilter over $\omega_{1}$ is regular. Benda and Ketonen [1] extended this further to show that Prikry's result actually follows from Kurepa's Hypothesis. Here we have the first inklings of the 'large cardinality nature' of nonregular ultrafilters over $\omega_{1}$ : it then follows immediately that $\omega_{2}$ must be inaccessible in $L$ whenever such ultrafilters exist. In this paper we shall show that $\omega_{1}$ itself must then be a very large cardinal in $L$.

We shall mainly work with the problem of $\left(\kappa, \kappa^{+}\right)$-regularity of ultrafilters over $\kappa^{+}$. A great deal of progress has been made in this area recently, the following being the main results:

1.3. THEOREM (BENDA AND KETONEN [1]). If $D$ is a non- $\left(\kappa, \kappa^{+}\right)$-regular ultrafilter over $\kappa^{+}$, then $D$ is a P-point, i.e. if $f: k^{+} \rightarrow \kappa^{+}$is unbounded $(\bmod D)$, then there is a set $X \in D$ such that for every $\alpha<\kappa^{+}$:

$$
\left|f^{-1}(\{\alpha\}) \cap X\right|<\kappa .
$$

1.4. Definition. Given two ultrafilters $D, U$ over a cardinal $\lambda$, say that $D$ is less than $U$ in the Rudin-Keisler order, in symbols, $D \leqslant_{\mathrm{RK}} U$, if there is a function $f: \lambda \rightarrow \lambda$ such that for any $X \subseteq \lambda$ :

$$
X \in D \leftrightarrow f^{-1}(X) \in U .
$$

In this case we also denote: $D=f_{*}(U)$. Similarly, given two functions $f, g$ : $\lambda \rightarrow \lambda$ say $f \leqslant_{\mathrm{RK}} g(\bmod D)$ if there is a function $h: \lambda \rightarrow \lambda$ so that $f=h \circ g$ $(\bmod D)$.

Hence, if $f \leqslant_{\mathrm{RK}} g(\bmod D)$, then $f_{*}(D) \leqslant_{\mathrm{RK}} g_{*}(D)$. For more on this order, see for example Kunen [10].

1.5. THEOREM (KANAMORI [5]). If $D$ is a non-( $\omega, \lambda)$-regular ultrafilter over a regular cardinal $\lambda$, then there is an ultrafilter $U$ below $D$ in the RudinKeisler order which extends the closed unbounded filter on $\lambda$.

Combining Theorems 1.3 and 1.5, we have:

1.6. THEOREM (KANAMORI [5]). If $D$ is a non- $\left(\kappa, \kappa^{+}\right)$-regular ultrafilter over $\kappa^{+}$, then $D$ has a first function $f: \kappa^{+} \rightarrow \kappa^{+}$; i.e., every function $<f(\bmod D)$ 
is bounded by a constant $<\mathrm{K}^{+}(\bmod D)$ and $f$ itself is not bounded by a constant $(\bmod D)$.

1.7. Definition. An ultrafilter $D$ over a cardinal $\lambda$ is weakly normal if every pressing down function (i.e., a function $f$ such that for any $\alpha>0: f(\alpha)<$ $\alpha)$ on $\lambda$ has range of cardinality $<\lambda$ on a set of $D$-measure 1 .

As a corollary to Theorem 1.6. we have:

1.8. THEOREM (KANAMORI [5]). If $D$ is a non- $\left(\kappa, \kappa^{+}\right)$-regular ultrafilter over $\kappa^{+}$, then there is a weakly normal ultrafilter below $D$ in the Rudin-Keisler order.

For weakly normal ultrafilters we have the following characterization of nonregularity:

1.9. THEOREM (KETONEN [7]). If $D$ is a weakly normal ultrafilter over a regular cardinal $\lambda$, then $D$ is $(\mu, \lambda)$-regular if and only if

$$
\{\alpha \mid c f(\alpha)<\mu\} \in D \text {. }
$$

Combining Theorems 1.8 and 1.9 , we get:

1.10. THEOREM (KANAMORI [5]). If $\kappa$ is singular, then every ultrafilter over $\kappa^{+}$is $\left(\kappa, \kappa^{+}\right)$-regular.

Here again we wish to point out the similarities with large cardinals: If $D$ is a $\lambda$-complete ultrafilter over $\lambda$, then it is a well-known result (Scott [15]) that there is an ultrafilter $U$ below $D$ which is actually normal: every pressing down function is constant $(\bmod U)$. In this connection we wish to note the following result which has an analogue (due to Scott [15]) in the measurable case.

1.11.THEOREM (BENDA AND KETONEN [1]). If there is a non- $\left(\kappa, \kappa^{+}\right)$-regular ultrafilter over $\kappa^{+}$, then

$$
2^{\kappa}=\kappa^{+} \rightarrow 2^{\kappa^{+}}=\kappa^{++} \text {. }
$$

1.12. Theorem (BENDA AND Ketonen [1]). (1) Suppose $f_{\alpha}: \lambda \rightarrow \mu(\alpha<\lambda)$ is a family of eventually different functions $(\bmod F)$, where $F$ is a $\lambda$-complete filter over $\lambda$. Then an ultrafilter $D \supseteq F$ is non- $(\mu, \lambda)$-regular if and only if the $f_{\alpha}$ are cofinal in the ultrapower of $\mu$.

(2) If there is a non- $\left(\kappa, \kappa^{+}\right)$-regular ultrafilter over $\kappa^{+}$, then $\kappa^{++}$is inaccessible in $L$.

Our methods in this paper are based on ideas of J. Silver [16] with an infusion of Benda-style techniques. Also, the paper of Vopěnka and Hrbácek [20] is relevant.

Our main results are the following:

1.13. THEOREM. If there is a uniform, non- $(\kappa, k+)$-regular ultrafilter over $\mathrm{K}^{+}$, then $0^{\#}$ exists. 
1.14. THEOREM. If there is a uniform, weakly normal ultrafilter over a regular cardinal $k$ which is non- $(\gamma, \kappa)$-regular for all $\gamma<\kappa$, then $0^{\#}$ exists.

Here we shall not bother to give the formal definition of $0^{\#}$; we only need a statement equivalent to the existence of $0^{\#}$.

1.15. Definition (Kunen [10]). Suppose $M$ is a transitive class model of ZFC and $k$ is a cardinal in $M$. Then $D$ is an $M$-ultrafilter on $\kappa$ if:

(I) $D$ is a proper subset of $P(k) \cap M$ containing no singletons.

(II) $\forall x, y: x \subseteq y \in P(\kappa) \cap M \wedge x \in D \rightarrow y \in D$.

(III) $\forall x \in P(\kappa) \cap M: x \in D$ or $k-x \in D$.

(IV) If $\eta<k$ and $\left\langle x_{\xi} \mid \xi<\eta\right\rangle \in M$ and each $x_{\xi} \in D$, then $\bigcap\left\{x_{\xi} \mid \xi<\eta\right\} \in M$.

(V) If $\left\langle x_{\xi} \mid \xi<k\right\rangle \in M$, then $\left\{\xi \mid x_{\xi} \in D\right\} \in M$.

The following result will then be used.

1.16. THEOREM (KUNEN [9]). $0^{\#}$ exists if and only if there is an L-ultrafilter $D$ over a cardinal $\lambda$ in $L$ such that every countable intersection of elements of $D$ is nonempty, if and only if there is an ultrafilter over some $P(\lambda) \cap L(\lambda$ a cardinal in $L$ ) such that the ultrapower of $L$ with respect to $D$ is well founded.

For more on $0^{\#}$, see Solovay [18].

By Theorem 1.8 , it clearly suffices to prove only 1.13 . In $\S 2$ we prove that under hypotheses of Theorem $1.13, K$ is weakly compact in $L$ and $\boldsymbol{K}^{+(L)}<\boldsymbol{k}^{+}$. Using these two facts, we then prove the existence of $0^{\#}$ in $\S 3$.

The author wishes to express his gratitude to Ronald Jensen, Aki Kanamori, Jack Silver and Menachem Magidor for many helpful discussions.

2. Weakly normal ultrafilters and the constructible universe. In the following, suppose that $D$ is a uniform ultrafilter over a regular cardinal $\lambda$.

2.1. Definition. (1) If $f, g: \lambda \rightarrow V$, then

$$
f \sim g \leftrightarrow\{\alpha \mid f(\alpha)=g(\alpha)\} \in D .
$$

(2) If $f: \lambda \rightarrow V$, then

$$
[f]_{D}=\{g \mid g \sim f \text { and } \forall h(h \sim f \rightarrow \operatorname{rank}(h) \geqslant \operatorname{rank}(g))\} .
$$

Here the rank of a set $x$ is the usual set-theoretic rank. We can now define the ultrapowers we need. Let $C$ be a transitive classmodel of set theory.

2.2. Definition. (1) The ultrapower of the class $C$ with respect to $D$ is the class $\Pi_{D} C=\left\{[f]_{D} \mid f: \lambda \rightarrow C\right\}$.

(2) The restricted ultrapower of the class $C$ with respect to $D$ is the class $\Pi_{D}^{*} C=\left\{[f]_{D} \mid f: \lambda \rightarrow C\right.$ and $\left.|\operatorname{range}(f)|<\lambda\right\}$.

(3) In either case, we define the $\epsilon$-relation on the ultrapower to be $[f]_{D} E[g]_{D} \leftrightarrow\{\alpha \mid f(\alpha) \in g(\alpha)\} \in D$. 
The idea of using a restricted ultrapower in set-theory appears in the paper of Vopěnka-Hrbácek [20]. It is actually a special case of Keisler's notion of a limit-ultrapower (see Keisler [6]). A word of caution: All the models which we will construct will most often be not well founded.

2.3. Proposition (KeIsler [6]). Define an embedding $i: C \rightarrow \Pi_{D}^{*} C$ by setting $i(x)=[x]_{D}$, the equivalence class of the constant function $x$, and let $j: \Pi_{D}^{*} C \rightarrow \Pi_{D} C$ be the map induced by the inclusion map. Then both $i, j$ are elementary embeddings, and they induce a commutative diagram.

Now assume that $D$ is weakly normal. Let $\lambda^{*}=[\lambda]_{D}$ and $\rho^{*}=[\mathrm{id}]_{D}$. The following result is esentially due to Vopenka and Hrbáček.

2.4. Proposition. The map $j$ is an onto map when restricted to $\lambda^{*}$ in $\Pi_{D}^{*} C:$

$$
j:\left\{[f]_{D}|| \mathrm{rng} f \mid<\lambda \text { and }[f]_{D} E \lambda^{*}\right\} \stackrel{\text { onto }}{\longrightarrow}\left\{[f]_{D}[f]_{D} E \rho^{*}\right\} \text {. }
$$

This is clear, since every function $<$ [id] $(\bmod D)$ has range of cardinality $<\lambda(\bmod D)$.

Thus we have an order-isomorphism via the map $j$ between the predecessors of $\lambda^{*}$ in $\Pi_{D}^{*} C$ and predecessors of $\rho^{*}$ in $\Pi_{D} C$. The 'ordinal' $\lambda^{*}$ gets mapped into a bigger ordinal than $\rho^{*}$ by $j$; thus in a sense $\lambda^{*}$ is the first ordinal moved. Here is the basic idea of Silver [16]: Well foundedness will be replaced by isomorphisms between structures. The next result is essentially due to Kunen [9] and in its present context to Silver [16]. It follows directly from Proposition 2.4 .

2.5. Proposition. Define a collection $U$ of 'subsets' of $\lambda^{*}$ as follows:

$$
U=\left\{[f]_{D} \mid \Pi_{D}^{*} C \vDash[f]_{D} \subseteq \lambda^{*} \text { and } \Pi_{D} C \vDash \rho^{*} E j[f]_{D}\right\} .
$$

Then the following statements hold:

I. If $x \in U$ then

$$
\Pi_{D}^{*} C|=| x \mid=\lambda^{*}
$$

II. For $x, y \in \Pi_{D}^{*} C$,

$$
\begin{aligned}
& \Pi_{D}^{*} C \vDash x \cup y=\lambda^{*} \rightarrow x \in U \text { or } y \in U, \\
& \Pi_{D}^{*} C \vDash x \cap y=0 \rightarrow x \notin U \text { or } y \notin U .
\end{aligned}
$$

III. If $F \in \Pi_{D}^{*} C$ and $\Pi_{D}^{*} C=F: \lambda^{*} \rightarrow \lambda^{*}$ is pressing down, then there is a $y \in \Pi_{D}^{*} C$ such that there is $a z \in U$ with

$$
\Pi_{D}^{*} C \vDash y<\lambda^{*} \wedge z=f^{-1}(\{y\}) .
$$

IV. If $F \in \Pi_{D}^{*} C$ and $\mathrm{II}_{D}^{*} C \vDash F: y \rightarrow V \wedge y<\lambda^{*}$ and for any a such that 
$\Pi_{D}^{*} C \vDash a<y$ we have $F(a) \in U$, then, if

$$
\Pi_{D}^{*} C \vDash z=\bigcap\{f(u) \mid u<y\},
$$

$z \in U$.

Thus, $U$ is a ' $\Pi_{D}^{*} C$-ultrafilter' in the sense of Definition 1.14 with the possible exception of the following property.

V. If $F \in \Pi_{D}^{*} C$ and $\Pi_{D}^{*} C \vDash F: \lambda^{*} \rightarrow V$, then there is a $Z \in \Pi_{D}^{*} C$ such that

$$
F(a) \in U \leftrightarrow \Pi_{D}^{*} C \vDash a E Z .
$$

To accomplish this, we need to extend the isomorphism of Proposition 2.4 to subsets of $\lambda^{*}$ in $\Pi_{D}^{*} C$. Some kind of 'smoothness' of the model $C$ is required. From now on we shall assume that $C$ is the constructible universe.

2.6. PROPOSITION. If $D$ is a weakly normal ultrafilter over $\lambda$, then there exists an isomorphism $G$ such that for any relation $R \subseteq \lambda \times \lambda$,

$$
G: \underset{\alpha<\lambda}{\prod_{D}}\left\langle L_{\alpha}, \epsilon, R \cap(\alpha \times \alpha)\right\rangle \cong \Pi_{D}^{*}\left\langle L_{\lambda}, \epsilon, R\right\rangle .
$$

2.7. Proposition. If $D$ is a weakly normal ultrafilter over $\lambda$, then there exists an isomorphism $H$ between the structure $\Pi_{D}^{*}\left\langle L_{\lambda^{+}}, \epsilon\right\rangle$, where $\lambda^{+}$is the (real) successor of $\lambda$, and an initial E-segment of the structure $\underset{\alpha<\lambda}{\prod_{D}}\left\langle L_{\alpha^{+}}, \epsilon\right\rangle$.

These two propositions are directly modeled after those of Silver [16] and their proofs are similar. The proof of Proposition 2.6 is a straightforward application of weak normality. For the sake of completeness, we shall include the proof of Proposition 2.7.

Proof of Proposition 2.7. Given an ordinal $\alpha<\lambda^{+}$, let $R^{\alpha} \subseteq \lambda \times \lambda$ be a (possibly nonconstructible) relation coding $\left\langle L_{\alpha}, \epsilon\right\rangle$; i.e., the structures $\left\langle\lambda, R^{\alpha}\right\rangle$ and $\left\langle L_{\alpha}, \epsilon\right\rangle$ are isomorphic. Then the set

$$
C^{\alpha}=\left\{\gamma \mid\left\langle\gamma, R^{\alpha} \cap(\gamma \times \gamma)\right\rangle \text { is an elementary substructure of }\left\langle\lambda, R^{\alpha}\right\rangle\right\}
$$

is a closed unbounded set and therefore belongs to $D$. Let $g^{\alpha}$ be a function so that for $\gamma \in C^{\alpha}, g^{\alpha}(\gamma)<\gamma^{+}$and

$$
\left\langle L_{g^{\alpha}(\gamma)}, \epsilon\right\rangle \cong\left\langle\gamma, R^{\alpha} \cap(\gamma \times \gamma)\right\rangle .
$$

We have by Proposition 2.6,

$$
\begin{aligned}
\Pi_{D}^{*}\left\langle L_{\alpha}, \epsilon\right\rangle & \cong \Pi_{D}^{*}\left\langle\lambda, R^{\alpha}\right\rangle \\
& \left.\cong \prod_{\boldsymbol{\gamma}<\lambda}\left\langle\gamma, R^{\alpha} \cap(\gamma \times \gamma)\right\rangle \cong \underset{\gamma<\lambda}{\prod_{D}\left\langle L_{g^{\alpha}}(\gamma)\right.}, \epsilon\right\rangle .
\end{aligned}
$$

Thus we have a canonical isomorphism for $\alpha<\lambda^{+}$

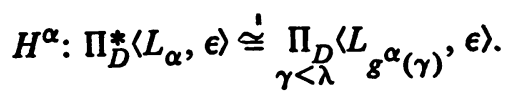


It remains to show that for $\alpha<\beta$,

$$
H^{\alpha}=H^{\beta} \text { on } \Pi_{D}^{*}\left\langle L_{\alpha}, \epsilon\right\rangle .
$$

This is an immediate consequence of the following fact: For any $\tau<\lambda^{+}$, let $P_{\boldsymbol{\tau}}$ be the isomorphism

$$
P_{\tau}:\left\langle\lambda, R^{\tau}\right\rangle \cong\left\langle L_{\tau}, \epsilon\right\rangle
$$

Then for any $\alpha<\beta$ the set

$$
\left\{\gamma \mid L_{\alpha} \cap P_{\beta}^{\prime \prime}(\{\delta \mid \delta<\gamma\})=L_{\alpha} \cap P_{\alpha}^{\prime \prime}(\{\delta \mid \delta<\gamma\})\right\}
$$

contains a closed unbounded subset of $\lambda$.

The functions $g^{\alpha}(\gamma)$ constructed in the above proof have an important property:

2.8. Proposition. If $\alpha<\beta<\lambda^{+}$, then there is a closed unbounded set $C \subseteq \lambda$ such that $\gamma \in C \rightarrow g^{\alpha}(\gamma)<g^{\beta}(\gamma)$.

Proof. If $\alpha<\beta<\lambda^{+}$, then there is an isomorphism $i$ from $\left\langle\lambda, R^{\alpha}\right\rangle$ onto a $R^{\beta}$-proper initial segment of the structure $\left\langle\lambda, R^{\beta}\right\rangle$. Hence there is an ordinal $\delta<\lambda$ s.t. $i(\gamma) R^{\beta} \delta$ for $\gamma<\lambda$. Let $C=\left\{\gamma \mid \gamma>\delta\right.$ and $i: \gamma \rightarrow \gamma$ and $\left.\gamma \in C^{\alpha} \cap C^{\beta}\right\}$. This set satisfies our requirements.

2.9. THEOREM. If $D$ is a weakly normal ultrafilter over $\lambda$ such that $D$ is not $(\gamma, \lambda)$-regular for any $\gamma<\lambda$, then there is an isomorphism

$$
H: \Pi_{D}^{*}\left\langle L_{\lambda^{+}}, \epsilon\right\rangle \cong \underset{\alpha<\lambda}{\prod_{D^{\prime}}\left\langle L_{\alpha^{+}}, \epsilon\right\rangle}
$$

extending the isomorphism $G^{-1}$ of Proposition 2.6.

Proof. By Proposition 2.7 it suffices to show that if the functions $g^{\alpha}\left(\alpha<\lambda^{+}\right)$are not cofinal $(\bmod D)$ in the ultraproduct

$$
\Pi_{D}\langle\dot{\alpha}+, \epsilon\rangle,
$$

then $D$ is $(\gamma, \lambda)$-regular for some $\gamma<\lambda$. If the $g^{\alpha}$ are not cofinal, there is a function $h: \lambda \rightarrow \lambda$ s.t. $h(\gamma)<\gamma^{+}$and for all $\gamma<\lambda$ and for all $\alpha<\lambda^{+}$we have: $g^{\alpha}<$ $h(\bmod D)$. Now, let $k_{\gamma}$ be a one-to-one function from $\lambda \rightarrow \lambda$ which maps $h(\gamma) \rightarrow \gamma$. Define for $\alpha<\lambda^{+}, \gamma<\lambda: h^{\alpha}(\gamma)=k_{\gamma}\left(g^{\alpha}(\gamma)\right)$. Each $h^{\alpha}$ is a pressing down function $(\bmod D)$. Therefore we may, without loss of generality, assume that there is a $\xi<\lambda$ such that $h^{\alpha} \leqslant \xi(\bmod D)$ for all $\alpha<\lambda^{+}$. But, by Proposition 2.8 , the functions $g^{\alpha}$ are mutually eventually different modulo the closed unbounded filter. Therefore, by Benda's Theorem 1.2, $D$ is $(\xi, \lambda)$-regular.

The following result is then a straightforward analog of Silver's Theorem 1.5 in [16]. 
2.10. THEOREM. If $D$ is not- $(\gamma, \lambda)$-regular for any $\gamma<\lambda$ and $D$ is weakly normal, then the ultrafilter $U$ satisfies condition $V$. so that

Proof. Suppose that $F \in \Pi_{D}^{*} C$ and $\Pi_{D}^{*} C \vDash F: \lambda^{*} \rightarrow V$. Let $y \in \Pi_{D} C$

$$
\Pi_{D} C \vDash y=\{s<\rho * \mid \rho * E(j F)(s)\}
$$

Then $H^{-1}(y)$ will satisfy the requirements of condition $V$.

The way is finally clear for large cardinality results:

2.11. THEOREM. If $D$ is weakly normal over $\lambda$ and $D$ is non- $(\gamma, \lambda)$-regular for any $\gamma<\lambda$, then $\lambda$ is $\Pi_{n}^{1}$-indescribable for every $n<\omega$ in $L$. That is, for any constructible relation $R$ on $\lambda, \Pi_{n}^{1}$ sentence $\dot{\phi}$ : If $\left(\langle\lambda,\langle, R\rangle \vDash \phi)^{L}\right.$, then there is an $\alpha<\lambda$ such that $(\langle\alpha,<, R \mid \alpha\rangle \vDash \phi)^{L}$. As a matter of fact,

$$
\left\{\alpha \mid(\langle\alpha,<, R| \alpha \dot{\prime} \vDash \phi)^{L}\right\} \in D .
$$

Proof. This is clear from Theorem 2.9, since $\phi$ becomes a first order statement in $L_{\lambda+}$.

Thus, we now know that $\lambda$ is weakly compact under the hypotheses of Theorem 2.11. We shall prove that $\lambda^{+(L)}<\lambda^{+}$in this situation by contradiction.

2.12. Proposition. If $X$ is a function $\lambda \rightarrow P(\lambda) \cap L$ with range of cardinality $<\lambda$, then

$$
[X] \in U \leftrightarrow\{\alpha \mid \alpha \in X(\alpha)\} \in D .
$$

This is immediate from the definition of $U$.

2.13. Proposition. For any sequence $\langle m(\alpha)| \alpha<\lambda$, where $m(\alpha)$ is a constructible subset of $\alpha$, there exists a function $A: \lambda \rightarrow P(\lambda) \cap L$ with range of cardinality $<\lambda$ such that

$$
\{\alpha \mid m(\alpha)=A(\alpha) \cap \alpha\} \in D \text {. }
$$

Proof. The 'set' $[m]_{D} \in \Pi_{D} L$. Let $A: \lambda \rightarrow P(\lambda) \cap L$ so that $H\left([A]_{D}\right)$ $=[m]_{D}$. Then $A$ satisfies our requirements. Note that by 2.9 , if $x \subseteq \lambda$ and $x \cap \alpha \in L$ for all $\alpha<\lambda$, then $x \in L$.

There is a useful modification of our basic construction: Instead of looking at all functions, we can restrict our attention to constructible functions: Define

$$
\begin{aligned}
\Pi_{D}^{L} L & =\left\{[f]_{D} \mid f \in L \wedge f: \lambda \rightarrow L\right\}, \\
\Pi_{D}^{* *} L & =\left\{[f]_{D}|f \in L \wedge f: \lambda \rightarrow L \wedge| \mathrm{mg}(f) \mid<\lambda\right\} .
\end{aligned}
$$

2.14. Proposition. If $\lambda^{+L}=\lambda^{+}$and $D$ is weakly normal and not $(\gamma, \lambda)$ regular for any $\gamma<\lambda$, then

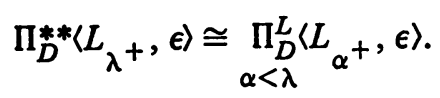


Proof. For $\alpha<\lambda^{+}$we can then require the relations $R^{\alpha}$ of Proposition 2.6 to be constructible. Theorem 2.9 then implies that

$$
\Pi_{D}^{*}\left\langle L_{\lambda^{+}}, \epsilon\right\rangle \cong \Pi_{\alpha<\lambda}\left\langle L_{\alpha^{+}}, \epsilon\right\rangle
$$

from which our claim follows since the $g^{\alpha}\left(\alpha<\lambda^{+}\right)$are then cofinal in the ultrapower.

2.15. Proposition. Under the hypotheses of Proposition 2.15: If $\left\langle X_{\xi}\right| \xi$ $\langle\lambda\rangle$ is a constructible sequence of subsets of $\lambda$, then there is a constructible function $A: \lambda \rightarrow P(\lambda) \cap L$ with range $<\lambda$ such that for any constructible $f: \lambda \rightarrow$ $\lambda$ with range $<\lambda$ we have

$$
\left\{\alpha \mid \alpha \in X_{f(\alpha)}\right\} \in D \leftrightarrow\{\alpha \mid f(\alpha) \in A(\alpha)\} \in D .
$$

PROoF. This follows immediately from the constructible analogues of Theorem 2.10 and Proposition 2.12.

Let $n$ be a positive integer. For a set $x,[x]^{n}$ denotes the set of all unordered $n$-tuples from the set $x$.

2.16. Proposition. Under the hypotheses of Proposition 2.15: If F: $[\lambda]^{n} \rightarrow\{0,1\}$ is constructible, then there is a function $X: \lambda \rightarrow \dot{P}(\lambda)$ with range of cardinality $<\lambda$ such that $X \in L$ and

(a) $\forall \alpha<\lambda, X(\alpha)$ is homogeneous for $F$; i.e., $F^{\prime \prime}[X(\alpha)]^{2}$ is a singleton.

(b) $\{\alpha \mid \alpha \in X(\alpha)\} \in D$.

Proof. Using the constructible analogue of Theorem 2.10 and standard techniques of, say, Kunen [10], we can show that there is a $[X]_{D} \in U \cap \Pi_{D}^{* *} L$ such that $\Pi_{D}^{* * *} L \vDash[X]_{D}$ is homogeneous for $i(F)$.

We shall present the proof of the entirely analogous 2.20 in more detail.

2.17. Definition. $F:[\lambda]^{n} \rightarrow \lambda$ is pressing down if for all $0<\alpha_{1}<\alpha_{2}$ $<\ldots<\alpha_{n}$,

$$
F\left(\left\{\alpha_{1} \ldots \alpha_{n}\right\}\right)<\alpha_{1} .
$$

2.18. THEOREM. Under the hypotheses of Proposition 2.14: If $F:[\lambda]^{n}$ $\rightarrow \lambda$ is a constructible pressing down function, then there is a set $X \in D \cap L$ and $a \xi<\lambda$ such that $F^{\prime \prime}[X]^{n} \subseteq \xi$.

Proof. Take $n=2$. For each $\alpha<\lambda$ define

$$
g_{\alpha}(\beta)=F(\{\alpha, \beta\}) \quad(\beta<\alpha) .
$$

Thus $g_{\alpha} \subseteq \alpha \times \alpha$. By the constructible analog of 2.14, there is a constructible map $T: \lambda \rightarrow \lambda \lambda$ with range of cardinality $\xi<\lambda$ so that for any $\alpha<\lambda, T(\alpha)$ is a pressing down function and 


$$
U=\left\{\alpha\left|g_{\alpha}=T(\alpha)\right| \alpha\right\} \in D \text {. }
$$

By a variant of Theorem 1.8 the set $Y=\left\{\alpha \mid c f^{L}(\alpha)>\left(\xi^{+}\right)^{L}\right\} \in D$. This can be seen for example as follows: Suppose that $Z=\left\{\alpha \mid c f^{L}(\alpha) \leqslant\left(\xi^{+}\right)^{L}\right\} \in D$. Then we can find a constructible sequence $\left\{A_{\alpha} \mid \alpha<\lambda\right\}$ of sets such that $L \vDash \forall \alpha<$ $\lambda: A_{\alpha} \subseteq \alpha$ has order type $\leqslant\left(\xi^{+}\right)^{L}$, and if $\alpha \in Z$, then $A_{\alpha}$ is cofinal in $\alpha$. But then, by Proposition 2.13, we can find a constructible $A \subseteq \lambda$ such that the set $\left\{\alpha \in Z \mid A_{\alpha}=A \cap \alpha\right\}$ has cardinality $\lambda$, contradiction by Theorem 2.11. Hence, if

$$
K(y)=\sup \{T(\alpha)(y) \mid \alpha<\lambda\},
$$

$K$ is pressing down on $Y$. By weak normality, there is a $\xi<\lambda$ so that

$$
Z=\{y \in Y \mid K(y) \leqslant \xi\} \in D \cap L .
$$

It then follows that $F \leqslant \xi$ on $[Z \cap U]^{2}$.

2.19. ThEOREM. Under the hypotheses of Proposition 2.15: Suppose that $\left\{f_{\alpha} \mid \alpha<\lambda\right\}$ is a constructible family of bounded functions $\lambda \rightarrow \lambda$. Then there is a constructible function $g: \lambda \rightarrow \xi(\xi<\lambda)$ such that for every $\alpha<\lambda$

$$
f_{\alpha} \leqslant \leqslant_{\mathrm{RK}} g(\bmod D \cap L) .
$$

Proof. Define a pressing down function $F$ by:

$$
F(\{\alpha, \beta\})=\left\{\begin{array}{l}
0 \text { if } \forall \gamma<\alpha<\beta: f_{\gamma}(\alpha)=f_{\gamma}(\beta), \\
\text { least } \gamma<\alpha \text { so that } f_{\gamma}(\alpha) \neq f_{\gamma}(\beta) \text { otherwise. }
\end{array}\right.
$$

By Theorem 2.20 we can find an $\eta<\lambda$ and a set $X \in D \cap L$ such that $F \leqslant \eta$ on $[X]^{2}$, i.e., for $\alpha, \beta \in X$ either $\forall \gamma<\alpha<\beta: f_{\gamma}(\alpha)=f_{\gamma}(\beta)$ or there is a $\mu<\eta$ such that $f_{\mu}(\alpha) \neq f_{\mu}(\beta)$. By Theorem 2.19 there is a constructible partitioning $\left\{Y_{\xi} \mid \xi<\theta\right\}(\theta<\lambda)$ such that $Y=U\left\{Y_{\xi} \mid \xi<\theta\right\} \in D, Y \subseteq X$ and for all $\xi<\theta$ either $\alpha, \beta \in Y_{\xi} \rightarrow \forall \gamma<\alpha<\beta: f_{\gamma}(\alpha)=f_{\gamma}(\beta)$ or $\alpha, \beta \in Y_{\xi} \rightarrow \exists \mu<\eta: f_{\mu}(\alpha) \neq$ $f_{\mu}(\beta)$. We can without a loss of generality assume that, for any $\xi<\theta,\left|Y_{\xi}\right|=\lambda$. This rules out the second possibility listed above since $\lambda$ is inaccessible in $L$. Hence, if we set $g=\xi$ on $Y_{\xi}$, then for $\gamma<\lambda$ :

$$
f_{\gamma} \leqslant_{\mathrm{RK}} g \text { on } Y-\boldsymbol{\gamma} \cdot \square
$$

2.20. COROllary. Under the hypotheses of Proposition 2.14: For every $\gamma<\lambda^{+}$there is $a \xi_{\gamma}<\lambda$ and a constructible function $g_{\gamma}: \lambda \rightarrow \xi_{\gamma}$ such that for any bounded function $h: \lambda \rightarrow \lambda$ with $h \in L_{\gamma}$ we have

$$
h \leqslant_{\mathrm{RK}} g_{\gamma}(\bmod D \cap L) .
$$

The following is a trivial modification of the fundamental result of Silver [16]. 
2.21. TheOREM (SILVER [16]). If $D$ is a weakly normal ultrafilter over $\lambda$ which is not $(\gamma, \lambda)$-regular for any $\gamma<\lambda$ such that

$$
\left.\Pi_{D}^{* *}\left\langle L_{\lambda}+(L), \epsilon\right\rangle \cong \underset{\alpha<\lambda}{\Pi_{D}^{L}\left\langle L_{\alpha}+(L)\right.}, \epsilon\right\rangle
$$

and

$$
\left|\Pi_{D}^{L} \omega\right|<\lambda
$$

then $\operatorname{cof}\left(\lambda^{+(L)}\right)<\lambda$.

We can finally prove:

2.22. THEOREM. If there is a weakly normal ultrafilter over $\lambda$ which is not $(\gamma, \lambda)$-regular for any $\gamma<\lambda$, then $\lambda^{+(L)}<\lambda^{+}$.

Proof. For suppose that $\lambda^{+(L)}=\lambda^{+}$. By Theorem 2.22: $\left|\Pi_{D}^{L} \omega\right| \geqslant \lambda$. By Corollary 2.21, there is a fixed $\xi<\lambda$ and constructible $g_{\gamma}: \lambda \rightarrow \xi\left(\gamma<\lambda^{+}\right)$ such that for any $f \in L_{\gamma}$

$$
f \leqslant_{\mathrm{RK}} g_{\gamma}(\bmod D \cap L) .
$$

Therefore

$$
\mid\left\{[f]_{D} \mid f \in L_{\gamma} \text { and } f: \lambda \rightarrow \omega\right\} \mid \leqslant\left(\xi^{+}\right)^{L} .
$$

Since $\lambda^{+(L)}=\lambda^{+}$by assumption and $\lambda$ is inaccessible in $L$,

$$
\left|\Pi_{D}^{L} \omega\right|<\lambda
$$

a contradiction.

3. The main results. We shall now prove our main results. As was remarked before, it suffices to prove Theorem 1.14.

From now on, assume that $D$ is a weakly normal ultrafilter over a regular cardinal $\lambda$ such that $D$ is not $(\gamma, \lambda)$-regular for any $\gamma<\lambda$. By the results of $\S 2$, $\lambda$ is inaccessible in $L$ and $\lambda^{+(L)}<\lambda^{+}$.

As before, for any $\tau<\lambda^{+}$pick $R^{\tau} \subseteq \lambda \times \lambda$ coding $\left\langle L_{\tau}, \epsilon\right\rangle$ and let $P_{\tau}$ : $\left\langle\lambda, R^{\tau}\right\rangle \rightarrow\left\langle L_{\tau}, \epsilon\right\rangle$ be the isomorphism. - Let $P=\left\{\tau \mid L_{\tau}<L_{\lambda^{+}}\right.$and $\left.\left.\tau\right\rangle\left(\lambda^{+}\right)^{L}\right\}$. As usual, the symbol ' $A<B$ ' means that $A$ is an elementary substructure of $B$. For $\tau \in P$, let

$$
B^{\tau}=\left\{\alpha \mid \alpha<\lambda,\left\langle\alpha, R^{\tau} \cap \alpha\right\rangle\left\langle\left\langle\lambda, R^{\top}\right\rangle \text { and } P_{\tau}^{\prime \prime}(\alpha) \cap \lambda=\alpha\right\} .\right.
$$

Taking the transitive collapse of $\left\langle\alpha, R^{\tau} \cap \alpha\right\rangle$, for $\alpha \in B^{\tau}$ we get an elementary embedding $\Pi_{\alpha}^{\tau}: L_{g^{\tau}(\alpha)} \rightarrow L_{\lambda}+$ such that $\Pi_{\alpha}^{\tau}(\alpha)=\lambda$ and $\alpha$ is the first ordinal moved. Here the functions $g^{\tau}$ are constructed as in the proof of Proposition 2.7. We can then define ultrafilters $U_{\alpha}^{\tau}$ over $P(\alpha) \cap L_{g} \tau_{(\alpha)}$ as follows:

$$
X \in U_{\alpha}^{\tau} \leftrightarrow \alpha \in \Pi_{\alpha}^{\tau}(X) .
$$


3.1. Lemma. (a) Let $\tau_{0}=\left(\lambda^{+}\right)^{L}$. Then

$$
X_{0}=\left\{\alpha \mid g^{\top} 0(\alpha)=\left(\alpha^{+}\right)^{L}\right\} \in D \text {. }
$$

(b) For any $\tau<\eta<\lambda^{+}, \tau, \eta \in P: g^{\tau}<g^{\eta}$ on a closed unbounded set.

(c) For any $f: \lambda \rightarrow \lambda$ such that $f(\alpha)<|\alpha|^{+}$there is a $\tau \in P$ such that $f \leqslant$ $g^{\tau}(\bmod D)$.

(d) For any $\tau<\eta<\lambda^{+}, \tau, \eta \in P$ : There is a closed unbounded set $C$ such that

$$
C \cap X_{0} \subseteq\left\{\alpha \mid U_{\alpha}^{\tau}=U_{\alpha}^{\tau}=U_{\alpha}^{\eta}\right\}
$$

Proof. To prove (a), use the fact that $\left(\lambda^{+}\right)^{L}<\lambda^{+}$and Theorem 2.9.

(b) is simply a restatement of Proposition 2.8. (c) follows immediately from the proof of Theorem 2.9 .

To prove (d), argue as follows: Given $\tau, \eta$, the set

$$
A=\left\{\alpha \mid L_{\tau} \cap P_{\eta}^{\prime \prime}(\{\delta \mid \delta<\gamma\})=L_{\eta} \cap P_{\eta}^{\prime \prime}(\{\delta \mid \delta<\gamma\})\right\}
$$

is closed unbounded. By (a), (b) there is a closed unbounded set $C \subseteq A$ so that on $C \cap X_{0}$

$$
\left(\alpha^{+}\right)^{L}<g^{\tau}(\alpha)<g^{\eta}(\alpha)
$$

Now, $L_{g^{\eta}(\alpha)}$ is the transitive collapse of $P^{\prime \prime}(\{\delta \mid \delta<\alpha\})$. Since all the subsets of $\alpha$ in $L_{g^{\eta}(\alpha)}^{g^{\eta}(\alpha)}$ appear already in $L_{g^{\tau}(\alpha)}$ for all $\alpha \in C \cap X_{0}, U_{\alpha}^{\tau}=U_{\alpha}^{\eta}$.

Thus, we can find ultrafilters $U_{\alpha}$ over $P(\alpha) \cap L$ for $\alpha \in X_{0}$ such that for every $\tau \in P$ there is a closed unbounded set $C^{\tau}$ such that for $\alpha \in X_{0} \cap C^{\tau}$ :

$$
X \in U_{\alpha} \leftrightarrow \alpha \in \Pi_{\alpha}^{\tau}(X) .
$$

To finish off the proof, by Theorem 1.16 it suffices to prove:

3.2. LEMmA. There is an $\alpha \in X_{0}$ such that the ultrapower

$$
\operatorname{Ult}\left(L, U_{\alpha}\right)=\Pi_{U_{\alpha}}^{L} L
$$

is well founded.

PROOF. If this was not the case, for every $\alpha \in X_{0}$ we can find a sequence $f_{i}^{\alpha}: \alpha \rightarrow$ ORD such that for all $i<\omega:$

$$
\left\{\gamma \mid \gamma<\alpha, f_{i}^{\alpha}(\gamma)>f_{i+1}^{\alpha}(\gamma)\right\} \in U_{\alpha}
$$

and each $f_{i} \in L_{\theta}$ for some ordinal $\theta$ depending only on $\alpha$. Form the elementary substructure $M_{\alpha}$ of $L_{\theta}$ generated by the set $\alpha \cup\left\{f_{i}^{\alpha} \mid i<\omega\right\}$. By collapsing $M_{\alpha}$, we get an ordinal $\beta<|\alpha|^{+}$such that

$$
\left\langle M_{\alpha}, \epsilon\right\rangle \cong\left\langle L_{\beta}, \epsilon\right\rangle \text {. }
$$

From this follows that we can without loss of generality assume that each $f_{i}^{\alpha}: \alpha \rightarrow|\alpha|^{+}$. Let $f$ be a function $\lambda \rightarrow \lambda$ such that $f(\alpha)<\left|\alpha^{+}\right|$and for all $\alpha, i$ : 


$$
f_{i}^{\alpha} \in L_{f(\alpha)}, \quad f(\alpha)>\left(\alpha^{+}\right)^{L} .
$$

By Lemma 3.1 there is a closed unbounded set $C$ and a $\tau \in P$ such that

$$
f(\alpha)<g^{\tau}(\alpha) \quad\left(\alpha \in C \cap X_{0}\right)
$$

and

$$
X \in U_{\alpha} \leftrightarrow \alpha \in \Pi_{\alpha}^{\tau}(X)
$$

where $\Pi_{\alpha}^{\tau}$ is an elementary embedding $L_{g^{\tau}(\alpha)} \rightarrow L_{\lambda}+$ with $\alpha$ the first ordinal moved.

But, given $\alpha \in C \cap X_{0}$ we then have

$$
\left(\Pi_{\alpha}^{\tau} f_{1}^{\alpha}\right)(\alpha)>\left(\Pi_{\alpha}^{\tau} f_{2}^{\alpha}\right)(\alpha)>\left(\Pi_{\alpha}^{\tau} f_{3}^{\alpha}\right)(\alpha)>\ldots,
$$

a contradiction.

\section{REFERENCES} 231-240.

1. M. Benda and J. Ketonen, On regularity of ultrafilters, Israel J. Math. 17 (1974),

2. C. C. Chang and H. J. Keisler, Model theory, North Holland, Amsterdam, 1973.

3. R. Jensen, Some combinatorial principles of $L$ (Mimeographed).

4. K. Devlin, Some weak versions of large cardinality axioms, Ann. Math. Logic 5 (1973), 291-326.

5. A. Kanamori, Ultrafilters over uncountable cardinals, Doctoral Dissertation, Univ. of Cambridge, 1975.

6. H. J. Keisler, Limit ultrapowers, Trans. Amer. Math. Soc. 107 (1963), 382-408. MR 26 \#6054.

7. J. Ketonen, Strong compactness and other cardinal sins, Ann. Math. Logic 5 (1972), 47-76.

8. Open problems in the theory of ultrafilters (to appear).

9. K. Kunen, Inaccessibility properties of cardinals, Doctoral Dissertation, Stanford Univ., 1968.

10. Some applications of iterated ultrapowers in set theory, Ann. Math. Logic 41 (1970), 179-227. MR 43 \#3080.

11. Ultrafilters and independents sets, Trans. Amer. Math. Soc. 172 (1972), 299-306. MR 47 \#3170.

12. K. Prikry, Changing measurable into accessible cardinals, Dissertationes Math. Rozprawy Mat. 68 (1970), 1-55. MR 41 \#6685.

13. - On a problem of Gillman and Keisler, Ann. Math. Logic 2 (1970), 179-187. MR $42 \# 4408$.

14. - On descendingly complete ultrafilters (to appear).

15. D. S. Scott, Measurable cardinals and constructible sets, Bull. Acad. Polon. Sci. Sér. Sci. Math. Astronom. Phys. 9 (1961), 521-524. MR 26 \#1263.

16. J. Silver, Indecomposable ultrafilters and $0^{\#}$ (to appear).

17. R. Solovay and D. Scott, Boolean-valued models for set theory, Proc. 1967 U.C.L.A. Summer Inst. (to appear).

18. R. M. Solovay, $A$ nonconstructible $\Delta_{3}^{1}$ set of integers, Trans. Amer. Math. Soc. 127 (1967), 50-75. MR 35 \#2748.

19. P. Vopěnka, An estimate of cardinality of a power, J. Symbolic Logic 35 (1970), 612. (Abstract).

20. P. Vopěnka and K. HrbáCek, On strongly measurable cardinals, Bull. Acad. Polon. Sci. Sér. Sci. Math. Astronom. Phys. 14 (1966), 587-591. MR 35 \#2747.

DEPARTMENT OF MATHEMATICS, UNIVERSITY OF HAWAII, HONOLULU, 